\title{
KILLING THE MIDDLE HOMOTOPY GROUPS OF ODD DIMENSIONAL MANIFOLDS
}

\author{
BY \\ C. T. C. WALL
}

The main object of this paper is to prove the theorem: If $W$ is an $m$ parallelisable $(2 m+1)$-manifold, whose boundary has no homology in dimensions $m, m+1$; then $W$ is $\chi$-equivalent to an $m$-connected manifold.

This is written as a sequel to Milnor's paper $A$ procedure for killing homotopy groups of differentiable manifolds. We attempt to preserve the notations of this paper, and refer to it as [M].

Milnor proves in [M] that $W$ is $\chi$-equivalent to an $(m-1)$-connected manifold, and we show in $\S 1$ that we can reduce $H_{m}(W)$ to a finite group. $\$ 2$ is devoted to the definition and study of a nonsingular bilinear form on this group, symmetric if $m$ is odd, and skew if $m$ is even. $\S 3$ applies these results to prove the theorem above, It follows, in the notation of [8], that $\Theta_{2 m}(\partial \pi)=0$ (this has also been proved by Milnor and Kervaire). In $\S 4$ we prove a more precise version of Milnor's reduction of $(m-1)$-parallelisable to $(m-1)$-connected manifolds; this is applied in $\S 5$ to obtain results about the topology of certain $(m-1)$-parallelisable $(2 m+1)$-manifolds. Our results are complete for a class of 5-manifolds, and yield an interesting test for cobordism.

Throughout this paper, "manifold" shall mean "compact connected differential manifold." Here, "differential" means "endowed with differential structure"; it seems a more suitable word for this concept than "differentiable," which ought to mean "admitting at least one differential structure."

1. Preliminaries. We consider manifolds $W$ of dimension $2 m+1$ (where $1<m)$. We suppose that $W$ is $m$-parallelisable, and that we have already killed the homotopy groups $\pi_{i}(W)$ for $i<m$; we will study the possibility of killing $\pi_{m}(W)$. Since $m<(1 / 2)$ dim $W$, every element of $\pi_{m}(W)$ is representable by an imbedding $f_{0}: S^{m} \rightarrow W$. The induced bundle $f_{0}^{*}\left(\tau^{2 m+1}\right)$ is trivial, so by Lemma 3 of $[\mathrm{M}]$ there exists an imbedding $f_{1}: S^{m} \times D^{m+1} \rightarrow W$ extending $f_{0}$. In this case we can carry out surgery without trouble; the only snag is that we are not sure of simplifying $\pi_{m}(W)$ when we do it.

We reconsider the proof of Lemma 2 of [M]. It is convenient to give it a somewhat different form. We first pass from $W$ to the manifold $W^{\prime \prime}$ obtained by removing the interior of $f_{1}\left(S^{m} \times D^{m+1}\right)$ from it, and then to the manifold $W^{\prime}$ obtained by glueing $D^{m+1} \times S^{m}$ in its place. It is easy to see that $\pi_{m}\left(W^{i}\right)$ $\rightarrow \pi_{m}(W)$ is onto, and its kernel is generated by the class of $f_{2}\left(e \times S^{m}\right)$, where $f_{2}: S^{m} \times S^{m} \rightarrow W^{\prime \prime}$ is induced by $f_{1}$ (and we use $e$ indiscriminately to denote an

Received by the editors July 21, 1961. 
unspecified base point). For the same reason, $\pi_{m}\left(W^{\prime \prime}\right) \rightarrow \pi_{m}\left(W^{\prime}\right)$ is onto, and its kernel is generated by the class of $f_{2}\left(S^{m} \times e\right)$. We will usually denote these two classes in $\pi_{m}\left(W^{\prime \prime}\right)$, or rather, the corresponding ones in $H_{m}\left(W^{\prime \prime}\right)$, by $z, x$.

We suppose, as in $\S 6$ of $[\mathrm{M}]$, that $H_{m+1}(\mathrm{Bd} W)$ and $H_{m}(\mathrm{Bd} W)$ vanish, hence so also does $H_{m-1}(\mathrm{Bd} W)$. The homology sequence for the pair $(W, \mathrm{Bd} W)$ and the Universal Coefficient Theorem, imply that $H_{m}(W)=H_{m}(W, \mathrm{Bd} W)$ and $H_{m+1}(W)=H_{m+1}(W, \mathrm{Bd} W)$ with any coefficient group, and similarly for cohomology, so in these dimensions Poincaré duality for $W$ has the same form as for a closed manifold.

We first consider the case when $H_{m}(W)$ has elements of infinite order.

Lemma 1. Let $x$ be of infinite order in $H_{m}(W)$ and be indivisible. Then if we perform surgery on $W$ starting from $x$, the new class $z$ in $H_{m}\left(W^{\prime \prime}\right)$ vanishes, so $H_{m}\left(W^{\prime}\right)$ is obtained from $H_{m}(W)$ by killing $x$.

Proof. Denote the chain $f_{1}\left(e \times D^{m+1}\right)$ by $\bar{q}$. Since $x$ is indivisible, by Poincaré duality there is a class $p \in H_{m+1}(W)$ with unit intersection number with $x$. Since $f_{1}\left(\left(S^{m}-e\right) \times D^{m+1}\right)$ is a cell, we may choose a representative cycle $\bar{p}$ for $p$ which avoids it: and clearly we may suppose that the only simplexes of $p$ contained in $f_{1}\left(S^{m} \times D^{m+1}\right)$ form $\bar{q}$, since the intersection number of $p$ with $x$ is unity. But now $\bar{q}-\bar{p}$ defines a chain in $W^{\prime \prime}$ whose boundary $\bar{z}=f_{2}\left(e \times S^{m}\right)$ determines $z$. Hence $z=0$ in $H_{m}\left(W^{\prime \prime}\right)$ and a fortiori also in $H_{m}\left(W^{\prime}\right)$.

Now as in $\S 6$ of [M], $W^{\prime}$ is $m$-parallelisable if $W$ is, so we can repeat the process to kill all elements of infinite order in $H_{m}(W)$. Hence we may assume $G=H_{m}(W)$ finite. Let its exponent (the 1.c.m. of the orders of its elements) be $\theta$. We shall take homology and cohomology with coefficient group $Z_{\theta}$, but still represent the classes by integral chains. Now $H_{m}\left(W, Z_{\theta}\right)=G$ by the Universal Coefficient Theorem; we shall identify these groups by this isomorphism. Consider the map $\bar{y} \rightarrow \partial \bar{y} / \theta$ of chain groups: this induces a homomorphism $\gamma: H_{m+1}\left(W, Z_{\theta}\right) \rightarrow H_{m}\left(W, Z_{\theta}\right)$ dual to the Bockstein in cohomology. This is onto since each element of the latter group has a representative $\theta$ times which is a boundary, and (1-1) since if $\bar{y}$ represents a class $y$ with $\gamma y=0$, there exists a chain $\bar{w}$ with $\partial \bar{w}=\partial \bar{y} / \theta$, and since $H_{m+1}(W)=0$ (by duality), $\bar{y}-\theta \bar{w}$, being a cycle, is a boundary, so $\bar{y}$ determines the zero element of $H_{m+1}\left(W, Z_{\theta}\right)$.

2. The nonsingular bilinear form. Combining $\gamma$ with isomorphisms deduced from Poincare duality and the Universal Coefficient Theorem we now have

$$
G=H_{m}(W) \cong H_{m}\left(W, Z_{\theta}\right) \cong H_{m+1}\left(W, Z_{\theta}\right) \cong H^{m}\left(W, Z_{\theta}\right)=\operatorname{Hom}\left(G, Z_{\theta}\right) .
$$

Hence we have a pairing of $G$ with itself to $Z_{\theta}$. Write $b: G \otimes G \rightarrow Z_{\theta}$.

Lemma 2. $b$ is a nonsingular bilinear form on $G$, symmetric if $m$ is odd and skew if $m$ is even. 
Proof. We have already proved the first part. For the second it is more convenient to work in cohomology (isomorphic to homology by the above). Here, $b$ is given by $b(x, y)=\beta x \cdot y$, evaluated on the fundamental class of $(W, \operatorname{Bd} W)$, where $\beta$ denotes the Bockstein. Now

$$
\begin{aligned}
b(x, y)+(-1)^{m} b(y, x) & =\beta x \cdot y+(-1)^{m} \beta y \cdot x \\
& =\beta x \cdot y+(-1)^{m} x \cdot \beta y \\
& =\beta(x y) .
\end{aligned}
$$

But $x y \in H^{2 m}\left(W, Z_{\theta}\right)$, so reverting to homology we get $H_{1}\left(W, \mathrm{Bd} W ; Z_{\theta}\right)$. But every element of this is the restriction of an integer class, so applying $\partial / \theta$ gives zero, as required.

Note. This result also follows by interpreting $b(x, y)$ as a linking number $(\bmod \theta)$.

We shall now show how the form $b$ determines the effect of surgery on $H_{m}(W)$. Let $x$ be the element chosen to operate on, and let $y$ be of order $r$ in $H_{m}(W)$. Since $r y=0, \theta \mid r b(y, x)$, so $(r / \theta) b(y, x)$ is an integer defined modulo $r$. (| denotes divisibility.) Represent $y$ by an $m$-cycle $\bar{y}$ not meeting $f_{1}\left(S^{m} \times D^{m+1}\right)$. In $W^{\prime \prime}, \bar{y}$ represents a homology class $y^{\prime}$, and $r y^{\prime}$ is a multiple of $z$.

LEMMA 3. If we write $r y^{\prime}=\lambda z$, we have $\lambda \equiv(r / \theta) b(y, x)(\bmod r)$.

Proof. Let $\bar{b}$ be an $(m+1)$-chain with $\partial \phi=r \bar{y}$. As in the proof of Lemma 1, if the intersection number of $\bar{p}$ and $x$ is $\lambda$, we may suppose that the only simplexes of $\bar{p}$ contained in $f_{1}\left(S^{m} \times D^{m+1}\right)$ form $\lambda \bar{q}$. Now $\bar{p}-\lambda \bar{q}$ defines a chain in $W^{\prime \prime}$, of boundary $r \bar{y}-\lambda \bar{z}$, hence $r y^{\prime}=\lambda z$. But as $(\partial / \theta)(\theta / r) \bar{p}=\bar{y}$, the class $\bmod \theta$ of $\theta \bar{p} / r$ corresponds under $\gamma$ to $y$, so by definition of $b$,

$$
b(y, x) \equiv(\theta \bar{r} / r) \cap x \equiv \theta \lambda / r(\bmod \theta)
$$

i.e.

$$
\lambda \equiv \frac{r}{\theta} b(y, x)(\bmod r) .
$$

Corollary. Let $b(y, x)=0$. Then there exists a class $y^{\prime \prime}$ in $H_{m}\left(W^{\prime \prime}\right)$ inducing $y$ in $H_{m}(W)$ and also of order $r$.

Proof. $r y^{\prime}=k r z$ for some integer $k$. We may choose $y^{\prime \prime}=y^{\prime}-k z$.

Before we can prove our main theorem we need a number-theoretic lemma about bilinear forms $b$.

LEMMA 4. Let $b: G \otimes G \rightarrow Z_{\theta}$ be a nonsingular bilinear form on the finite Abelian group $G$. Write $c(x)$ for $b(x, x)$.

(i) If $b$ is symmetric and $c(x)=0$ for all $x$, then $\theta=2$ and we can find $a$ basis $\left\{x_{i}, y_{i}: 1 \leqq i \leqq r\right\}$ for $G$ such that

$$
b\left(x_{i}, y_{j}\right)=\delta_{i j} b\left(x_{i}, x_{j}\right)=b\left(y_{i}, y_{j}\right)=0 \text {. }
$$


(ii) If $b$ is skew-symmetric, we can find elements $x_{i}, y_{i}$ of order $\theta_{i}$ in $G$ $(1 \leqq i \leqq r)$ such that

$$
\begin{aligned}
b\left(x_{i}, x_{j}\right) & =b\left(x_{i}, y_{j}\right)=b\left(y_{i}, y_{j}\right)=0 \text { for } i \neq j ; \\
c\left(x_{i}\right) & =0, \quad b\left(x_{i}, y_{i}\right) \text { has order } \theta_{i},
\end{aligned}
$$

and $G$ contains the direct sum of the cyclic subgroups generated by the $x_{i}, y_{i}$ as a direct summand of index at most 2.

COROLlARY. Under the conditions of (ii), if $B$ is the subgroup generated by the $x_{i}$, then either

$$
G \cong B \oplus B \text { or } \quad G \cong B \oplus B \oplus Z_{2} .
$$

Proof. (i) Under these hypotheses, for all $x, y$ in $G$,

$$
2 b(x, y)=b(x, y)+b(y, x)=c(x+y)-c(x)-c(y)=0 .
$$

Hence the exponent of $G$ is 2 . We now pick $x_{i}, y_{i}$ by induction. Choose any nonzero $x_{1}$, then since $b$ is nonsingular there exists $y_{1}$ with $b\left(x_{1}, y_{1}\right)=1$. Since $c\left(x_{1}\right)=0, y_{1} \neq x_{1}$. Now $G$ is the direct sum of the subgroup $G_{p}\left\{x_{1}, y_{1}\right\}$ and $H$, the annihilator of $G p\left\{x_{1}, y_{1}\right\}$, and $b$ induces a nonsingular form on $H$, so we may continue the induction. (All this is of course well known.)

Note. If $x_{1}, x_{2}, \cdots$ belong to a group, $G p\left\{x_{1}, x_{2}, \ldots\right\}$ denotes the subgroup which they generate.

(ii) Since $b$ is skew, $c(x)=b(x, x)=-c(x)$, so has order 2. Moreover, $c(x+y)-c(x)-c(y)=b(x, y)+b(y, x)=0$, so $c$ is a homomorphism $G \rightarrow Z_{2}$. Now since $G$ is a finite Abelian group it is the direct sum of its Sylow subgroups $S_{p}$, and these are clearly orthogonal under $b$, so we can take them separately.

First, suppose $p$ odd. Let $x_{1}$ be an element of maximal order $p^{r}$ in $S_{p}$. Then since $b$ is nonsingular there exists $y_{1}$ such that $b\left(x_{1}, y_{1}\right)$ has order $p^{r}$. Then $y_{1}$ has order $p^{r}$ (not greater, since this was maximal) and $G$ contains the direct sum of the cyclic groups generated by $x_{1}, y_{1}$; for if $0=\lambda x_{1}+\mu y_{1}$, then

$$
0=b\left(\lambda x_{1}+\mu y_{1}, y_{1}\right)=\lambda b\left(x_{1}, y_{1}\right)+\mu c\left(y_{1}\right)=\lambda\left(b x_{1}, y_{1}\right)
$$

so $\lambda$ is divisible by $p^{r}$; similarly, so is $\mu$. Again we have $G=G p\left\{x_{1}, y_{1}\right\} \oplus H$, where $H$ is the annihilator of $x_{1}, y_{1}$, since any $z \in G$ can be written as

$$
z=b\left(z, y_{1}\right) x_{1}-b\left(z, x_{1}\right) y_{1}+h
$$

with $h \in H$. $b$ induces a nonsingular form on $H$, so we may apply induction to obtain our theorem.

For $p=2$ we apply the same argument, if $1<r$. The proof of independence of $x_{1}, y_{1}$ must be modified as follows. By the equation above, $b\left(x_{1}, y_{1}\right)$ has order at most 2 , so $\lambda$ is divisible by $2^{r-1}$, so by 2 . Similarly, so is $\mu$. Hence $\mu c\left(y_{1}\right)=0$, and we may proceed as before. (The modification of the direct sum argument 
is left to the reader.) We may suppose that $c\left(x_{1}\right)=0$, for if not, and $c\left(y_{1}\right)=0$, we interchange $x_{1}, y_{1}$; whereas if $c\left(x_{1}\right)=c\left(y_{1}\right) \neq 0$, we may replace $x_{1}$ by $x_{1}+y_{1}$.

Finally, suppose $G$ has exponent 2. If the order of $G$ is two, $G$ has the required form. If it is greater, let $x_{1}$ be any nonzero element of $\operatorname{Ker} c$, and $y_{1}$ such that $b\left(x_{1}, y_{1}\right) \neq 0$; then we can split off the direct summand $G p\left\{x_{1}, y_{1}\right\}$ as before. This concludes the proof.

Note. (i) We can be somewhat more precise in our reduction of $(G, b)$, but this is of no advantage for the applications we shall make of the lemma.

(ii) The above proof is complicated by the possibility $c \neq 0$ in (ii). We shall show in $\$ 5$ that for $m$-parallelisable $W, c$ must in fact vanish.

\section{Proof of theorem.}

Theorem. Let $W$ be $m$-parallelisable, of dimension $2 m+1$. If the boundary of $W$ has no homology in dimensions $m, m+1, W$ is $\chi$-equivalent to an $m$-connected manifold.

Proof, $m$ even. By Theorem 3 of $[\mathrm{M}]$, we may suppose $W(m-1)$-connected, and by Lemma $1, H_{m}(W)$ finite. By Lemma 2 it admits a nonsingular skew form $b$, so by Lemma 4 , we may express $G$ in the special form there given. First suppose $B$ is not zero. Take the class $x_{1}$, represent by a sphere, and perform surgery. Then $H_{m}\left(W^{\prime \prime}\right)$ is generated by elements $x_{i}^{\prime}, y_{i}^{\prime}, z$; where $x_{i}^{\prime}, y_{i}^{\prime}$ are classes mapping to $x_{i}, y_{i}$ in $H_{m}(W)$, for uniformity of notation we have denoted the generator of the "extra" $Z_{2}$ in $G$ (if there is one) by $x_{0}$, and $x_{1}^{\prime}, z$ are the classes of $f_{2}\left(S^{m} \times e\right), f_{2}\left(e \times S^{m}\right)$. By the corollary to Lemma 3 , we may suppose that for $i \neq 1, x_{i}^{\prime}, y_{i}^{\prime}$ have the same orders as $x_{i}, y_{i}$. Also by Lemma 3 , we may choose $y_{1}^{\prime}$ such that $\theta_{1} y_{1}^{\prime}=-z$, and since $c\left(x_{1}\right)=0, \theta_{1} x_{1}^{\prime}$ $=\lambda \theta_{1} z$, for some integer $\lambda$.

Suppose if possible $\lambda \neq 0$. Then in $W^{\prime}, x_{1}^{\prime}$ becomes zero, so we have (using primes to denote corresponding elements)

$$
\theta_{1} y_{1}^{\prime \prime}=-z^{\prime}, \quad \lambda \theta_{1} z^{\prime}=0
$$

so $y_{1}^{\prime \prime}$ has order $\lambda \theta_{1}^{2}$. The orders of other basic elements are unchanged from $G$, and there are no new ones. We see that the resulting group fails to have the form required by the corollary to Lemma 4 . Hence $\lambda=0$. Then in $W^{\prime}$ we have $\theta_{1} y_{1}^{\prime \prime}=-z^{\prime}$, and $y_{1}^{\prime \prime}$ has infinite order. By Lemma 1 , we may now kill $y_{1}^{\prime \prime}$, and we have then simplified the finite group $G$. Hence by induction we may simplify till $G$ is 0 or $Z_{2}$. In the latter case perform surgery starting with the nonzero element $x$ of $G$. Then $2 x^{\prime}=\lambda z$ for some odd $\lambda$. Hence $H_{m}\left(W^{\prime}\right)$ is cyclic of some odd order, which by Lemma 4 must be unity, so in this case also we can make $W m$-connected.

We must now consider the case when $m$ is odd. The main difference from the earlier case is that there (using Lemma 4) the effect of surgery was already determined by the choice of the class $x$. But for $m$ odd there is the additional question of product structure for $S^{m} \times S^{m}$. Now $H^{m}\left(S^{m} \times S^{m}\right)$ is the 
free Abelian group on two generators induced from the projections on the factors. Any autohomeomorphism of $S^{m} \times S^{m}$ induces an automorphism of this group and so a linear transformation of determinant \pm 1 . We represent this by the appropriate matrix

$$
\left(\begin{array}{ll}
a & b \\
c & d
\end{array}\right)
$$

over $Z$. If $m \neq 1,3,7$, there is no element of Hopf invariant odd in $\pi_{2 m+1}\left(S^{m+1}\right)$ and so no map $S^{m} \times S^{m} \rightarrow S^{m}$ with both degrees odd (by [2;5]). Hence $a b, c d$ are even, i.e., $a, d$ have the opposite parity to $b, c$. However,

LEMMA 5. $S^{m} \times S^{m}$ admits diffeomorphisms corresponding to any matrix

$$
\left(\begin{array}{ll}
a & b \\
c & d
\end{array}\right)
$$

which is unimodular, and with $a, d$ of opposite parity to $b, c$.

Proof. This falls naturally into two parts. First we produce a diffeomorphism for the matrix

$$
\left(\begin{array}{rr}
1 & 0 \\
2 & -1
\end{array}\right)
$$

and then prove that this, together with the trivially representable matrices

$$
\left(\begin{array}{rr} 
\pm 1 & 0 \\
0 & \pm 1
\end{array}\right) \text { and }\left(\begin{array}{rr}
0 & \pm 1 \\
\pm 1 & 0
\end{array}\right)
$$

generates the group of all matrices satisfying the conditions above. We define the diffeomorphism using a map of Hopf [5]. Let $(p, q) \in S^{m} \times S^{m}$. Then draw the great circle through the points $p, q$ of $S^{m}$, and let $q^{\prime}$ be the other point of it at the same distance from $p$ as $q$ is. Thus if $q$ is $p$ or its antipode, $q^{\prime}=q$ is unique. Then consider the map $S^{m} \times S^{m} \rightarrow S^{m} \times S^{m}$ defined by $(p, q) \rightarrow\left(p, q^{\prime}\right)$. It is clearly (1-1) and infinitely differentiable (and its own inverse), and since $m$ is odd it corresponds to the matrix

$$
\left(\begin{array}{rr}
1 & 0 \\
2 & -1
\end{array}\right)
$$

as promised.

Alternatively we may use a map $f: S^{m} \rightarrow S O_{m+1}$ of index 2 (it is well known that such exist), and define a diffeomorphism by $F(x, y)=(x, f(x) \cdot y)$ : this corresponds to the matrix

$$
\left(\begin{array}{ll}
1 & 2 \\
0 & 1
\end{array}\right)
$$


The proof about generators for the group parallels Kuroš [6, Appendix B]. The only change is where he sets $a=q c+a^{\prime}, 0 \leqq a^{\prime}<c$, we must put $a=2 q^{\prime} c+a^{\prime \prime},-c<a^{\prime \prime} \leqq c$. But $a^{\prime \prime}=c$ is impossible, as this would imply that $a$ had the same parity as $c$. The remainder of the proof is unaltered (working with

$$
\left(\begin{array}{ll}
1 & 2 \\
0 & 1
\end{array}\right)
$$

which is easily expressed by the matrices above). In fact the corresponding projective group, a subgroup of index 3 in the modular group, is $Z_{2} * Z$.

Proof of theorem, $m$ odd. First suppose $c(x)$ not identically zero. Choose $x$ such that $c(x) \neq 0$. We represent $x$ by an $S^{m}$ and perform surgery. We shall adhere to our earlier notation, denoting corresponding classes with primes. We consider the elements $x^{\prime}, z$ of $H_{m}\left(W^{\prime \prime}\right)$. By Lemma $3, r x^{\prime}=s z$, say, where $r$ is the order of $x$, and $r \nmid s$ since $c(x) \neq 0$. Hence the h.c.f. $(r, s)=h<r$. Set $r=r^{\prime} h, s=s^{\prime} h$. Choose $\lambda, \mu$ such that $\lambda r^{\prime}+\mu s^{\prime}=1$ : we may suppose $\lambda, \mu$ of opposite parity since if they are both odd, $r^{\prime}, s^{\prime}$ must be of opposite parity and we may take $\lambda+s^{\prime}, \mu-r^{\prime}$.

Write $y=\lambda z+\mu x^{\prime}$. Since $\lambda, \mu$ have opposite parity, by Lemma 5 we may choose the product structure in $S^{m} \times S^{m}$ so that $y$ corresponds to one of the factors. Then glue in $D^{m+1} \times S^{m}$ to kill $y$ and give $W^{\prime}\left({ }^{1}\right)$. Now in $W^{\prime}$,

$$
r x^{\prime \prime}=s z^{\prime} \quad \text { i.e., } h\left(r^{\prime} x^{\prime \prime}-s^{\prime} z^{\prime}\right)=0
$$

and $0=y^{\prime}=\lambda z^{\prime}+\mu x^{\prime \prime}$, so

$$
\begin{aligned}
x^{\prime \prime} & =\left(\lambda r^{\prime}+\mu s^{\prime}\right) x^{\prime \prime}=\lambda\left(r^{\prime} x^{\prime \prime}-s^{\prime} z^{\prime}\right), \\
z^{\prime} & =\left(\lambda r^{\prime}+\mu s^{\prime}\right) z^{\prime}=-\mu\left(r^{\prime} x^{\prime \prime}-s^{\prime} z^{\prime}\right),
\end{aligned}
$$

hence the group generated by $x^{\prime \prime}, z^{\prime}$ has order a factor of $h$ (in fact equal to it) which is less than $r$. Since the index of this group in $H_{m}\left(W^{\prime}\right)$ equals that of $G p\{x\}$ in $H_{m}(W)$, (for $G p\left\{x^{\prime}, z\right\}$ contains the kernels of both $H_{m}\left(W^{\prime \prime}\right)$ $\rightarrow H_{m}(W)$ and $\left.H_{m}\left(W^{\prime \prime}\right) \rightarrow H_{m}\left(W^{\prime}\right)\right)$ we have succeeded in decreasing the order of $H_{m}(W)$, or more precisely, in replacing it by a divisor of itself.

We may repeat the above process as long as $c$ is not zero. Hence by induction ( $G$ being finite) we may suppose $c=0$, and $(G, b)$ as in (i) of Lemma 4 . Perform surgery on the class $x_{1}$. If $A$ denotes the subgroup of $G$ generated by $x_{i}, y_{i}$ for $1<i \leqq r$, then by Lemma 3 , and corollary, $H_{m}\left(W^{\prime \prime}\right)$ $=A \oplus G p\left\{x_{1}^{\prime}, y_{1}^{\prime}, z\right\}, 2 x_{1}^{\prime}$ is an even multiple of $z$, and we may suppose $2 y_{1}^{\prime}=z$.

Write $2 x_{1}^{\prime}=(4 k+d) z$, where $d=2$ or 4 , and kill $x_{1}^{\prime}-2 k z$. Then $d z^{\prime}=0$, and so $H_{m}\left(W^{\prime}\right)=A \oplus G p\left\{y_{1}^{\prime \prime}\right\}$, and $y_{1}^{\prime \prime}$ has order $2 d$.

(1) This form of surgery is rather more general than that used in [M], but it follows from our proof of Lemma 5 that it is equivalent to a series of the spherical modifications of $[M]$. 
Write $U$ for $W^{\prime}, u$ for $y_{1}^{\prime \prime}$. Then since $b$ is nonsingular, and $u$ is the only basis element of $H_{m}(U)$ of order greater than 2, the order of $c(u)$ equals the order of $u$. Now perform surgery starting with the class $u$. Then in $H_{m}\left(U^{\prime \prime}\right)$ we have $2 d u$ equal to an odd multiple of the new class $w$. Then we kill $u$ (we have no need to worry about the product structure this time), and

$$
H_{m}\left(U^{\prime}\right)=A \oplus Z_{k} \quad \text { if } k \text { is the odd order of } w .
$$

(The sum is direct as $A$ is a 2-group.) But now, by the first part of the proof, we can replace the order of the group by a divisor of itself such that the new group has the form of Lemma 4 (i), and so has order not exceeding that of $A$. Hence in the second case also we have succeeded in decreasing the order of $H_{m}(W)$, so our induction is complete, and we may reduce the group to zero.

COROLlaRy. Let $T^{2 m}$ be a homotopy sphere which bounds a $\pi$-manifold. Then it bounds a contractible manifold.

For the result is trivial if $m=1$, and otherwise we may apply the theorem to find an $m$-connected manifold with boundary $T$. But by relative Poincaré duality, such a manifold must be contractible.

Complement. Let $T^{4 m}$ be a homotopy sphere, and $W$ a $\pi$-manifold with boundary $T$. Then there is a contractible manifold $C$ with boundary $T$, such that if $W^{\prime}$ is formed by glueing $W$ to $C$ along $T$, there is a parallelisable manifold $M$, with boundary $W^{\prime}$.

Proof. Our construction of $C$ from $W$ by surgery was by choosing at each stage a class on which to perform the construction. By Lemma 5 of $[\mathrm{M}]$, if we choose the correct trivialisation of the normal bundle at each stage, the manifolds $\omega(W, f)$ are parallelisable: this goes also for the proof of Theorem 2 of $[\mathrm{M}]$. Since the trivialisations given for the tangent bundles of these manifolds fit together on the boundary, we may form $M$ by glueing these manifolds together, and it will then be parallelisable.

These results are of use for computing the groups $\Theta_{m}$ of $J$-equivalence classes of homotopy spheres. Our reference is [7]. In the notation of those notes, the ábove corollary states $\Theta_{2 m}(\partial \pi)=0$. Since Milnor proves that $\Theta_{2 m} / \Theta_{2 m}(\partial \pi)$ is finite, it follows that for each $m, \Theta_{2 m}$ is a finite group. Also, using other results of Milnor, $\Theta_{4}$ and $\Theta_{12}$ vanish. We may also show $\Theta_{6}=0$, and will sketch the proof (we omit details since a simpler proof is known). By Thom [8], the spinor cobordism group in dimension 6 is isomorphic to the stable homotopy group $\pi_{n+6}(M(\operatorname{Spin} n))$. Results of Adams [1] relate these to a spectral sequence which starts with

$$
\operatorname{Ext}_{A_{2}}^{* *}\left(H^{*}\left(M(\operatorname{Spin} n), Z_{2}\right), Z_{2}\right)
$$

where $A_{2}$ denotes the Steenrod algebra mod 2. A straightforward computation of this in low dimensions now shows that the group in question vanishes. 
Hence a homotopy 6-sphere, being a spin manifold, bounds another, $W$ say. But $W$ is a spin manifold, and so 3-parallelisable, and the result now follows by the theorem above.

All these results have been obtained independently by M. Kervaire (including a stronger form of the above complement), and will appear in a joint paper by M. Kervaire and J. Milnor entitled Groups of homotopy spheres, which will also contain the substance of [7]. Recent results of Smale and Munkres have emphasised the importance of the groups $\Theta_{m}$.

4. Simplifying certain $(m-1)$-parallelisable $(2 m+1)$-manifolds. Suppose that $U$ is an $(m-1)$-parallelisable $(2 m+1)$-manifold, and in addition that $H_{m-1}(U)$ is torsion free, hence free Abelian. By Theorem 3 of [M], $U$ is $\chi$-equivalent to an $(m-1)$-connected manifold. We wish to obtain a slight refinement of this result. Now since $H_{m-1}(U)$ is free, $H^{m}(U, A)$ $=\operatorname{Hom}\left(H_{m}(U), A\right)$. The obstruction $p$ to $m$-parallelisability of $U$ lies in $H^{m}\left(U, \pi_{m-1}(O)\right)$, where $O$ denotes the stable orthogonal group. We make the convention of regarding $p$ as a function on $H_{m}(U)$.

We may now state the reduction lemma.

Lemma 6. If $U$ is a compact $(m-1)$-parallelisable $(2 m+1)$-manifold, with $H_{m-1}(U)$ torsion free, then there is a sequence of surgeries taking $U$ to an $(m-1)$ connected manifold $U^{*}$, and such that

(i) If $m>2$, there are induced isomorphisms of $H_{m}(W), H_{m+1}\left(W, Z_{\theta}\right)$ at each stage, which commute with the Bockstein operator, with intersection numbers $\bmod \theta$, and with $p$.

(ii) If $m=2$, there are forwards maps of $H_{2}(W)$ at each stage, inducing isomorphisms of its torsion subgroup, and backwards maps of $H_{3}\left(W, Z_{\theta}\right)$, commuting with the same three invariants, and inducing isomorphisms

$$
H_{2}(U)=H_{2}\left(U^{*}\right), \quad H_{3}\left(U^{*}, Z_{\theta}\right)=H_{3}\left(U, Z_{\theta}\right) .
$$

Proof. If $m<2$, we can take $U^{*}=U$ (supposed connected).

(i) If $m>2$, we may first use the procedure of [M] to kill successively the $\pi_{i}(U): 0<i<m-1$. We note that this induces natural isomorphisms of $H_{m}(W), H_{m+1}\left(W, Z_{\theta}\right)$ at each stage, and if the resulting manifold is $U_{1}$, $H_{m-1}\left(U_{1}\right)$ is naturally imbedded in $H_{m-1}(U)$, hence it also is torsion free. Since $m-1>1$, by the Hurewicz isomorphism, $\pi_{m-1}(U)=H_{m-1}(U)$, so is free Abelian. Now since $U_{1}$ is $(m-1)$-parallelisable, by construction, we may kill the generators in turn: it is easy to see that $H_{m}(W)$ and $H^{m+1}\left(W, Z_{\theta}\right)$ remain unaltered. The required commutativities now follow from the naturality of the several invariants for the successive inclusion maps $W^{\prime \prime} \rightarrow W$ and $W^{\prime \prime} \rightarrow W^{\prime}$.

(ii) If $m=2$, we may first choose elements of $\pi_{1}(U)$ inducing generators of $H_{1}(U)$, and kill these as before. Hence we may assume $H_{1}(U)=0$. We now select a set of generators of $\pi_{1}(U)$ and kill them in order. At each stage, we have exact sequences 


$$
\begin{aligned}
& 0 \rightarrow B_{2}\left(W^{\prime \prime}\right) \rightarrow B_{2}\left(W^{\prime}\right) \rightarrow Z \rightarrow 0 \\
& \begin{array}{c}
\mathbb{R} \\
H_{2}(W)
\end{array} \\
& 0 \rightarrow Z_{\theta} \rightarrow H_{3}\left(W^{\prime \prime}, Z_{\theta}\right) \rightarrow H_{3}\left(W, Z_{\theta}\right) \rightarrow 0 \text {. } \\
& \mathbb{R} \\
& H_{3}\left(W^{\prime}, Z_{\theta}\right)
\end{aligned}
$$

Let the resulting manifold be $U_{1} . H_{2}(U)$ is contained in $H_{2}\left(U_{1}\right)$ with free Abelian quotient group. We lift a set of generators of this quotient group to $H_{2}\left(U_{1}\right)$ : we may suppose that $p$ vanishes on each. For if $U$ is 2-parallelisable, by Theorem 3 of $[\mathrm{M}]$, we may suppose that $U_{1}$ is also, so $p$ vanishes identically; yet if not, $p$ is a nonzero homomorphism $H_{2}(U) \rightarrow Z_{2}$, and to each lifted generator on which $p$ does not vanish we may add an element of $H_{2}(U)$ with the same property.

Since $p$ vanishes on these generators, they are representable by imbeddings of $S^{2} \times D^{3}$, and we may perform $\chi$-constructions to kill them. At each stage of this process we have exact sequences (by Lemma 1)

$$
\begin{gathered}
0 \rightarrow Z \rightarrow H_{2}\left(W^{\prime \prime}\right) \rightarrow H_{2}\left(W^{\prime}\right) \rightarrow 0, \\
\mathbb{R} \\
H_{2}(W) \\
0 \rightarrow H_{3}\left(W^{\prime \prime}, Z_{\theta}\right) \rightarrow H_{3}\left(W, Z_{\theta}\right) \rightarrow Z_{\theta} \rightarrow 0 . \\
\mathbb{R} \\
H_{3}\left(W^{\prime}, Z_{\theta}\right)
\end{gathered}
$$

The resulting manifold is the required $U^{*}$. We have exhibited maps of the homology groups as stated, which induce isomorphisms as stated (this is clear for $H_{2}$ and will follow by duality for $H_{3}$ ). From the diagrams above, and from the naturality of the invariants for the inclusion maps, follow again the various commutation relations.

Corollary. Suppose in addition that the boundary of $U$ has no homology in dimensions $m, m+1$, so that a bilinear form can be set up as in \$2. Then the transition from $U$ to $U^{*}$ preserves the bilinear form.

This is clear, since the form is defined by Bocksteins and intersection numbers.

5. Topology of certain $(m-1)$-parallelisable $(2 m+1)$-manifolds, $(m$ even). We may now apply the above lemma to make our manifolds $(m-1)$-connected, and the methods of the rest of this paper will then apply. We shall study the homomorphism $c$ of Lemma 4 (ii), and show in particular that if $W$ is $m$-parallelisable, then $c=0$.

We shall suppose in the following that $W$ satisfies the condition:

(A) $W$ is a compact $(m-1)$-parallelisable $(2 m+1)$-manifold, such that $H_{m-1}(W)$ is torsion free and $H_{m}(W)$ finite, and the boundary of $W$ has no homology in dimensions $m, m+1$; where $m$ is even. 
The obstruction $p$ to $m$-parallelisability has coefficient group $\pi_{m-1}(0)$, which was evaluated by Bott [3] as $Z$ if $m=0(\bmod 4)$; as 0 if $m=6(\bmod 8)$; and as $Z_{2}$ if $m=2(\bmod 8)$. But under $(\mathrm{A}), H^{m}(W)$ vanishes, so $p=0$ unless $m=2(\bmod 8)$, when the coefficient group is $Z_{2}$.

Lemma 7. If $W$ satisfies (A), $x \in H_{m}(W)$ and $p(x)=0$, then $c(x)=0$.

Proof. By Lemma 6, we may suppose $W(m-1)$-connected. Note that $p(x)=0$ is the condition that $x$ be representable (by an imbedding of $S^{m} \times D^{m+1}$ ). We take a base of $H_{m}(W)$ as in Lemma 4. Let $y_{1}$ be an element of this base of order greater than 2 with $p\left(y_{1}\right)=0, c\left(y_{1}\right) \neq 0$. We shall deduce a contradiction.

Let $2 n$ be the order of $y_{1}$ (it is even since $c\left(y_{1}\right) \neq 0$ ). Let $A$ be the subgroup of $H_{m}(W)$ generated by $x_{j}, y_{j}$ for $j \neq 1$. Since $y_{1}$ is representable, we can perform surgery. As in the proof of the theorem, using Lemma 3, we have $H_{m}\left(W^{\prime \prime}\right)=A \oplus G p\left\{x_{1}^{\prime}, y_{1}^{\prime}, z\right\}$ where $2 n x_{1}^{\prime}=z, 2 n y_{1}^{\prime}=(2 \lambda n+n) z$. Hence $H_{m}\left(W^{\prime}\right)=A \oplus G p\left\{x_{1}^{\prime \prime}, z^{\prime}\right\}$ and this last group is cyclic of order $\geqq 2 n>2$, which contradicts the corollary to Lemma 4 ( $A$ being of the type admitted by that corollary).

Now suppose that $H_{m}(W)$ contains an element $x$ for which $p(x)=0$, $c(x) \neq 0$. Let $M_{2 \theta}$ be obtained from $S^{m} \times S^{m+1}$ by performing surgery on $2 \theta$ times a generator of $H_{m}\left(S^{m} \times S^{m+1}\right)$. Clearly, $M$ satisfies (A). It is easy to see that $H_{m}(M)=2 Z_{2 \theta}$, and since $p=0$ for $S^{m} \times S^{m+1}$, by [M] we may suppose that it is 0 for $M$, hence $c=0$, since by what we have already proved $c$ vanishes on each generator. Let $x_{0}$ be a generator of $H_{m}(M)$ (of order $2 \theta$ ).

Form $W \# M$. Now $H_{m}(W \# M)=H_{m}(W) \oplus H_{m}(M)$, and it is clear that $b$ admits the direct sum decomposition and $c$ and $p$ are additive. Consider the element $x+x_{0}$ of order $2 \theta$. We have

$$
p\left(x+x_{0}\right)=p(x)+p\left(x_{0}\right)=0, \quad c\left(x+x_{0}\right)=c(x)+c\left(x_{0}\right)=c(x) \neq 0 .
$$

By the proof of Lemma 4, an odd multiple $y$ of $x+x_{0}$ can be chosen as a basis element of $H_{m}(W \# M)$; this will have order greater than 2 , and $p(y)=0$, $c(y) \neq 0$, which contradicts what we proved above. This proves the lemma.

The lemma may be rephrased: $c=p$ or $c=0$. For if the kernel of $c$ properly contains that of $p$, which has index at most 2, the kernel of $c$ is the whole group, so $c=0$. If $m \neq 2(\bmod 8)$, this simply states $c=0$. If $m=2(\bmod 8)$, we shall now show that whether $c$ is $p$ or 0 depends only on $m$. In fact we shall produce a closed manifold $V$ satisfying (A), and with $p(V) \neq 0$. Form $W \# V$. $p$ and $c$ are additive. There are now two cases.

If $c(V)=p(V), c(W \# V)=c(W)+c(V) \neq 0$ since $c(V) \neq 0$. Hence it equals $p(W \# V)=p(W)+p(V)$, and we deduce $c(W)=p(W)$.

If $c(V)=0, c(W)+c(V) \neq p(W)+p(V)$ since $c(V) \neq p(V)$. Hence $c(W)$ $=c(W \# V)=0$.

The manifold $V$ may be constructed as follows. Take the nontrivial $S^{m+1}$ 
bundle $U$ over $S^{m}$ (defined since $m=2(\bmod 8)$ ). Let $x$ generate $H_{m}(U)$. $p(2 x)=2 p(x)=0$, so we may perform surgery and kill $2 x$. This yields a manifold $V$ which satisfies (A), and $x$ determines a class $x^{\prime}$ in $V$ with $p\left(x^{\prime}\right) \neq 0$.

In the case $m=2$, we can show that $c=p$. (We have not yet succeeded in deciding the question in any other cases.) For the Wu manifold $P(1,2)$ (see [4]) satisfies (A) and has $H_{m}(P)=Z_{2}$. Since $b$ is nonsingular, $c \neq 0$. We may sum up these results as

Proposition 1. Let $W$ satisfy (A). If $m \neq 2(\bmod 8), c(W)=p(W)=0$. If $m=2(\bmod 8)$, there is an integer $r_{m} \bmod 2$ such that $c(W)=r_{m} p(W)$ for all $W$. Moreover, $r_{2}=1$.

Now for $m=2, p$ is the second Stiefel class $w^{2}$. For any closed 5-manifold $W$ satisfying (A), we know $c$ by elementary homology theory, and may now use Wu's formulae to deduce from $w^{2}$ the operation of the Steenrod squares in $W$.

We finally turn to the problem of deciding when in Lemma 4 (ii) there is an extra $Z_{2}$. Since $c$ is a homomorphism and $b$ nonsingular, $G$ has an element $y_{0}$ with $c(x)=b\left(x, y_{0}\right)$ for all $x$. It is easy to show that the extra $Z_{2}$ appears if and only if $c\left(y_{0}\right) \neq 0$. For 5 -manifolds, this fact admits an interesting interpretation. We know that $c=p=w^{2}$. Now we have the commutative diagram

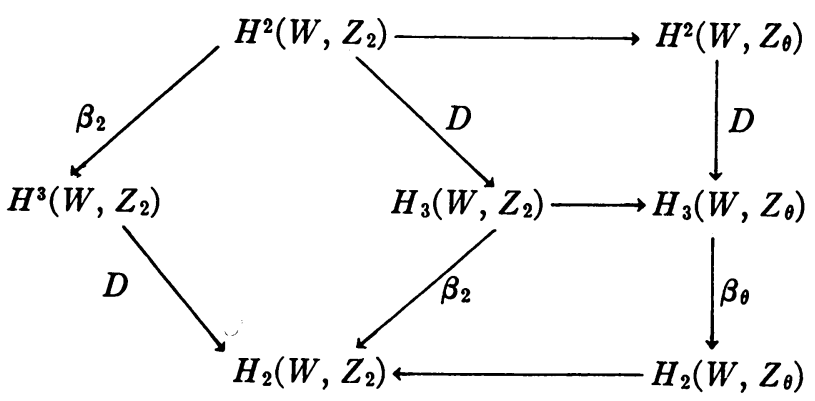

where $D$ denotes duality isomorphisms, $\beta_{2}$ is the Bockstein, and the horizontal maps are induced by the obvious homomorphisms of coefficient groups. But $w^{2} \in H^{2}\left(W, Z_{2}\right)$ maps under $\beta_{2}$ to $w^{3} \in H^{3}\left(W, Z_{2}\right)$, and $c \in H^{2}\left(W, Z_{2}\right)$ maps to $y_{0} \in H_{2}\left(W, Z_{\theta}\right)$, so each of $w^{2} w^{3}, c\left(y_{0}\right)$ is equal to the Kronecker product of $c$ with its image in $H_{2}\left(W, Z_{2}\right)$. Now since a closed oriented 5 -manifold $W$ is cobordant to zero if and only if the Stiefel number $w^{2} w^{3}[W]$ vanishes by [8], we have proved

Proposition 2. Let $W$ be a closed oriented 5-manifold such that $H_{1}(W)$ is torsion free, $\mathrm{H}_{2}(W)$ finite. Then there exists a finite Abelian group $B$ such that either
(i) $H_{2}(W)=B \oplus B$
or
(ii) $H_{2}(W)=B \oplus B \oplus Z_{2}$.

$W$ is cobordant to zero if and only if (i) holds. 


\section{REFERENCES}

1. J. F. Adams, On the structure and applications of the Steenrod algebra, Comment. Math. Helv. 32 (1958), 180-214.

2. - On the non-existence of elements of Hopf invariant one, Bull. Amer. Math. Soc. 64 (1958), 279-282.

3. R. Bott, An application of the Morse theory to the topology of Lie groups, Proc. Internat. Congr. Math. Edinburgh, 1958.

4. A. Dold, Erzeugende der Thomachen Algebra N, Math. Z. 65 (1956), 25-35.

5. H. Hopf, Über die Abbildungen von Sphären auf Sphären niedrigerer Dimension, Fund. Math. 25 (1935), 427-440.

6. A. G. Kurosh, Theory of groups, Vol. II (Trans. K. A. Hirsch), Chelsea, New York, 1955. 1959.

7. J. Milnor, Differentiable manifolds which are homotopy spheres, Princeton notes, Spring,

M. - A procedure for killing homotopy groups of differentiable manifolds, Proc. Symposium Tucson, Arizona, 1960.

8. R. Thom, Quelques proprietes globales des varietés differentiables, Comment. Math. Helv. 28 (1954), 17-86.

Trinity College,

Cambridge, England 